\title{
The Outcome of a Week of Intensive Cognitive Stimulation in an Elderly Care Setup: A Pilot Test
}

\author{
Roxana Agrigoroaie and Adriana Tapus
}

\begin{abstract}
In the context of a worldwide aging population, it is important to find solutions to help the elderly maintain their cognitive functions. This research was done in the context of the ENRICHME. We investigate the outcome of a 5 day intensive cognitive stimulation with an elderly individual. Each day was composed of two sessions (one at 11am, and one at $3 \mathrm{pm})$. During each session the participant played three cognitive games (i.e., digit cancellation, integer matrix task, Stroop game), two of them having two difficulty levels. The mood of the participant was also recorded before and after each interaction session. Evidence was found that even after a few sessions, the performance of the participant increased for all games. The performance of each game and each difficulty level was analyzed based on the interaction time (11am or $3 \mathrm{pm})$ and the interaction day. A detailed analysis of the performance is presented together with a discussion of these results.
\end{abstract}

\section{INTRODUCTION}

The worldwide population is growing older [1]. A lot of research is dedicated to finding viable solutions for enabling the elderly to stay independent for longer in their own home environments, and to enhance their quality of life. One such solution for providing personalized care is represented by the socially assistive robots (SAR) [2].

Some such robots have been developed in the past few years [3], [4], [5]. They have been developed as it was shown that they can be used to stimulate cognitive functions [6], [7] and they can provide monitoring [3]. The aim of the HOBBIT European project [4] was to use a robot in order to visually observe and interpret the actions of the elderly so that appropriate assistive services can be provided. The RAMCIP European project [5] focuses on how a robot can monitor the behaviour of an elderly individual with mild cognitive impairment in order to provide assistance in case of need. The Robot-Era ${ }^{1}$ [8] robotic system was designed as a mobile personal service robot with the aim of helping the elderly in their daily-life activities and to stimulate mental and physical exercise. The MoveCare ${ }^{2}$ European research project developed a robotic system capable of providing with the help of artificial intelligence assistance and transparent monitoring for the elderly in their own homes.

Another research project for the elderly with mild cognitive impairment (MCI) is represented by the $\mathrm{ENRICHME}^{3}$

\footnotetext{
*This work was funded and done in the context of the EU H2020 ENRICHME projet, Grant Agreement No:643691

The authors are at the Autonomous Systems and Robotics Laboratory, U2IS, ENSTA-ParisTech, Université Paris-Saclay, Palaiseau, France firstname. lastname@ensta-paristech.fr

${ }^{1}$ http://www.robot-era.eu

${ }^{2}$ www.movecare-project.eu

${ }^{3}$ www.enrichme.eu
}

EU Horizon 2020 project. Its purpose is to develop a personal socially assistive robot that can enhance the everyday life of the elderly and to help them stay independent for longer. The robot adapts its behavior based on the user's profile, past experiences, cognitive disability level, emotional state, and other preferences. The profile of the user is determined based on multiple psychological questionnaires (e.g., Eysenck Personality Questionnaire, Morningness-Eveningness Questionnaire).

One important requirement of the ENRICHME system is to provide cognitive stimulation that is adapted for the elderly with MCI. For this purpose, multiple cognitive games have been developed specifically for this purpose. There are multiple platforms that provide cognitive games (e.g., Luminosity $^{4}, \mathrm{M}^{3} \mathrm{~W}^{5}$, Sociable ${ }^{6}$ ), but none of them could be customized based on the elderly profile, preferences and robot's requirements. For each game that we developed, there is real time feedback, both written and spoken by the robot, and a customized feedback is provided at the end of the game. The variation of the performance is shown for the previous 6 days when exiting each game. This feature enables the elderly to see how they are progressing in each game.

As the ENRICHME system adapts based on the profile of the individual it interacts with, first it had to be investigated how the profile of an individual can be determined. For this purpose, multiple questionnaires have been used in our previous studies [9], [10] to determine which aspects of these questionnaires are of importance. We found evidence that the personality, neuroticism, empathy level, morningnesseveningness type, sensory profile, are all important traits that have to be taken into account.

The current study investigates how the cognitive performance of an elderly individual with mild cognitive impairment (MCI) varies after a week long intensive cognitive stimulation scenario. More specifically, the participant had to complete two cognitive sessions each day (one in the morning at around $11 \mathrm{am}$, and one in the afternoon at around $3 \mathrm{pm}$ ) for 5 days. The results of the first day are not considered for the analysis, as the influence of the novelty factor (the presence of the new experimenter and new difficulty levels for the games) could have an influence on the results. Of interest for this research is how the performance differs between morning and afternoon, and over time. The participant was already familiar with the robot, as he is

\footnotetext{
${ }^{4}$ www.luminosity.com

${ }^{5}$ https://m3w-project.eu/

${ }^{6} \mathrm{http}: / /$ www.cognitivetraining.eu/
} 


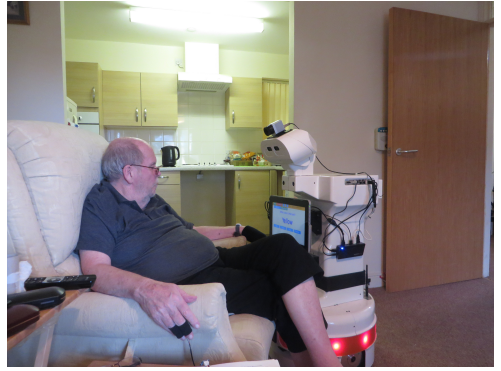

Fig. 1: Experimental setup

one of the users that tests the ENRICHME system for a longer period of time (i.e., 12 weeks). The participant is also familiarized with the cognitive games, but only with the easy levels and not with the difficult ones.

The paper is structured as follows. Section II presents the experimental design. We detail the scenario, present the robotic platform used, and describe the participant in more details. The cognitive games tested are presented in Section III. The results are summarized in Section IV. In Section $\mathrm{V}$, a discussion of the results is provided, while the paper concludes with Section VI.

\section{EXPERIMENTAL DESIGN}

\section{A. Robotic platform and sensors}

The robotic platform of the ENRICHME project is the TIAGo robot[11], developed by PAL Robotics ${ }^{7}$ in Spain. The robot was customized based on the requirements of the research project.

It features a mobile base, a lifting torso, a touch-screen mounted on the torso, a head that incorporates an Orbbec Asus RGB-D camera. An Optris PI450 thermal camera was mounted on the head of TIAGo (see Figure 1).

The data from the two cameras (i.e., RGB-D and thermal), together with the data from a Grove $\mathrm{GSR}^{8}$ were recorded for physiological data analysis. The GSR sensor was positioned on the ring and middle fingers of the right hand. The participant wore a touch-screen glove on its left hand to ease the usage of the touch-screen.

\section{B. Questionnaires}

For this study, the participant had to complete the following questionnaires:

Eysenck Personality Questionnaire (EPQ) [12] was developed to measure four personality traits: extraversion, psychotism, neuroticism, and lie. The Eysenck model proposes that each of these four traits has an underlying physiological basis. For this study of importance are the personality traits of extraversion (which is based on cortical arousal [13]), and neuroticism (which Eysenck considered it to be equivalent to the degree of emotionality [14]). Each trait is measured on a scale from 1 to 12 (e.g., individuals with scores lower than 6 are considered introverted, while individuals with scores higher than 6 are considered extraverted).

\footnotetext{
${ }^{7}$ www.pal-robotics.com

${ }^{8}$ www.seeed.cc/Grove-GSR_Sensor
}

Morningness - Eveningness Questionnaire (MEQ) [15] is widely used in the literature to assess if a person is of a morning type or an evening type. It was shown [15] that evening individuals perform better cognitively in later hours of the day, than morning individuals. This questionnaire was used in order to understand whether being a morning type or an evening type can affect the cognitive performance.

Reinforcement Sensitivity Theory - Personality Questionnaire (RST-PQ) was developed by Corr [16] to determine the personality of an individual as defined by the Reinforcement Sensitivity Theory (RST)[17]. The model, which is a neuroscience model of personality, states that the personality of an individual is based on three neurobehavioral systems (Behavior Activation System, Behavior Inhibition System, Fight-Flight-Freeze System). As the Eysenck model, which was the starting point of RST, RST relates all personality traits to underlying physiological basis.

Positive And Negative Affect Schedule (PANAS) [18] measures both positive and negative affect. Each of its 20 items are measured using a 5-point Likert scale. High values of possitive affect are associated with emotions like enthusiasm, or alertness, while low values for possitive affect is a characteristic of sadness. On the other hand, high values for negative affect are indicators of distress or unpleasurable engagement, while low values for negative affect represent the absence of any feeling [19].

Custom developed post-questionnaires contains a series of questions to assess how the participant rated each interaction. Moreover, a 5 point Likert scale was used so that the participant could rate the following items: difficulty of each game, stressfullness of each game, length of each game, utility of robot/tablet feedback, etc.

\section{Scenario}

The experiment presented in this study took place in the house of an elderly participant, who is one of the testing users of the ENRICHME system. The house is part of a housing facility designed for the elderly from the United Kingdom. The interaction took place in the living room of the participant (see Figure 1).

Originally, the experiment was planned for two participants, both end user testers of the ENRICHME system. However, due to the degradation of the visual capabilities of one of the participants, he had to be removed from this study. As a result, the experiment was carried out with only one participant. From the original 3 days of interaction with each participant, it resulted a 5 day interaction with only one of them.

The interaction took place two times each day, a session in the morning (at around 11am), and the second session in the afternoon (at around 3pm). For the rest of this study, we consider the afternoon session as an evening session.

As the participant was not familiar with the experimenter, two days of visits were planned. In the first day, the experimental setup was put in place. While on the second day, the two interaction sessions took place. The data on this day was not considered for the analysis as we wanted to eliminate all 
influences of different novelty parameters (e.g., presence of new experimenter, the experiment, the difficult levels of the cognitive games).

Each of the 8 sessions considered for the analysis consisted of three main phases:

- Relaxation: we wanted the participant to feel relaxed before performing the cognitive activities. Furthermore, this relaxation part enabled us to determine the baseline for the GSR and to see variations in GSR between different times of day.

- Cognitive games: the participant had to play 5 cognitive games. The order of the games was chosen by the participant, and each game had to be played only once. A description of each game is presented in Section III.

- News Reading: as a continuation of our previous study [20], we tested the news reading application with two interaction distances and different sensory stimuli (either only visual, or a combination of visual and auditory).

Before the relaxation phase and after the news reading phase, the participant filled the PANAS questionnaire. After each interaction session, the participant had to also fill in the custom developed post-questionnaire.

\section{Participant description}

The participant is a 69 years old male from the United Kingdom. He is a retired self employed decorator. He used to live alone, but he moved to the housing facility after he fell and he broke his hip. Not very long ago, he injured his right shoulder, and even though he is right handed he had to use his left hand for interacting with the touchscreen of the robot. He has no previous knowledge about robotics, except the news that he sees on the television. He has been smoking for more than 40 years and he likes to drink coffee. He does not drink any alcohol.

The results from the questionnaires show that he is very extraverted (a score of 12 out of 12), and that he has a low neuroticism level (a score of 2 out of 12), he is an intermediate type when considering the morningness eveningness questionnaire, with a score of 56. The MEQ score can be between 16 and 86 . Evening types have scores between 16 and 41 ; morning types have scores between 59 and 86 .

His results from the RST-PQ show that his FFFS score is above average (3.3 out of 4 ), while his behavior inhibition score is very low (1.1 out of 4$)$. Regarding the behavior activation system, for 2 of the 4 subscales (reward interest, and impulsivity) he scored below average ( 2.1 and 2.3 out of 4 ), while for the other two (reward reactivity and goal driven potential) he scored above average (2.6 and 3.1 out of 4 ).

\section{Cognitive Games}

The ENRICHME system offers a series of nine cognitive games (i.e., digit cancellation, letter cancellation, integer matrix task, decimal matrix task, memory game, hangman, puzzle, Stroop game, speed game), each with multiple difficulty levels. Out of these, three (see Figure 2) have been chosen for this study (i.e., digit cancellation, integer matrix task, and the Stroop game).

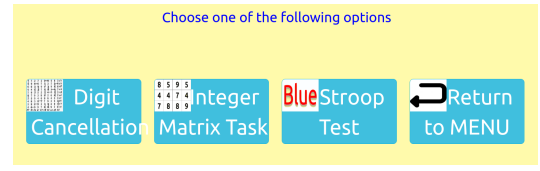

Fig. 2: Cognitive games

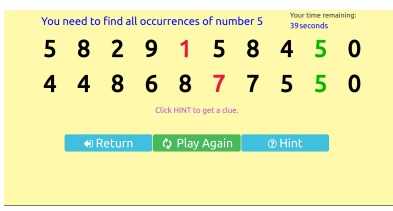

(a)

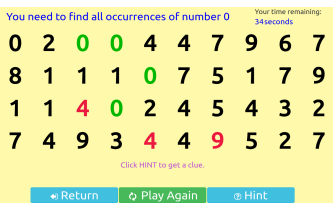

(b)
Fig. 3: Digit Cancellation: (a) Easy level; (b) Difficult level

These have been chosen as they exercise the following cognitive functions: attention, speed, mental arithmetic. Below is a description of each of the games.

\section{A. Digit Cancellation}

In this game, the user is presented with a list of a certain length which contains shuffled digits. In the given time (i.e., 50 seconds), the user has to find all occurrences of a randomly chosen digit in the given list. The color of a correctly chosen item is changed to green, while for a wrong answer it turns red. For this study two difficulty levels were developed. For the easy level the length of the list is equal to 20 (see Figure 3a), while for the difficult level the length of the list is equal to 40 (see Figure $3 \mathrm{~b}$ ). The game ends either when the user finds all the occurrences, or when the time is up. After the game a feedback is given with the number of mistakes made and the total game time.

\section{B. Integer Matrix Task}

In this game the user is shown a matrix with digits. The goal is to find the two digits whose sum equals 10. Feedback is given after each selection. Once the matrix is correctly solved, a new one is presented. If the user makes a mistake, the same matrix is shown until the correct answer is found. The final goal is to solve as many matrices as possible in a total time of 5 minutes. Two difficulty levels (see Figure 4) were developed: easy (Figure 4a) (matrix size of $2 \times 2$ ), and difficult (Figure 4b) (matrix size of $4 \times 4$ ).

\section{Stroop}

For this game, a variation of the original word-color Stroop test [21] was developed. The user has to press on the button

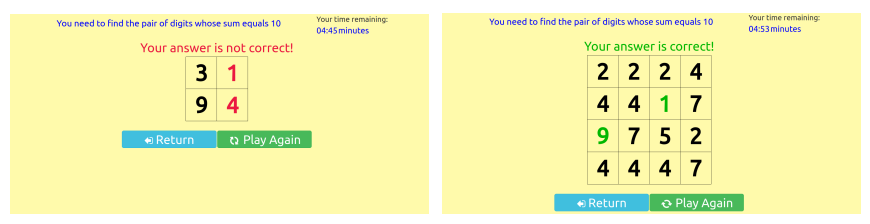

(a)

(b)

Fig. 4: Matrix Task: (a) Easy level; (b) Difficult level 


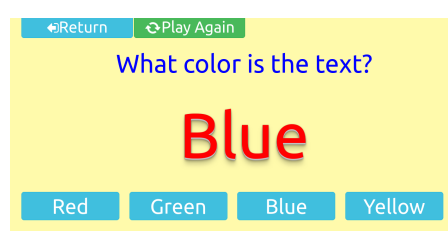

Fig. 5: Stroop Game trial example

which corresponds to the color of the word written on the screen ("What color is the text?"). The game was developed with four possible colors: Red, Green, Blue, and Yellow. For each button press a feedback is given. There is no time limit, but the user has to find the right answer as quickly as possible. Before each stimulus was shown, a warning signal was given in the form of a fixation phase of 1 second. The purpose of this phase was to prepare the participant for the stimulus and to lead to a reduction of the reaction time [22].

For this research the number of total trials of this game was set to 50. In this game there were two types of trials: congruent, and incongruent. In a congruent trial the color of the text is the same as the color depicted by the word written on the screen (e.g., the word Blue written in blue color). For the incongruent trial the color of the text was not the same as the color depicted by the word (e.g., the word Blue written in red color as shown in Figure 5).

\section{Results}

In this section, we present the results corresponding to the performance during each of the cognitive games and the results of the PANAS questionnaire. As the participant could choose the order of the games, we noticed that the participant preferred to start with the easy levels of each game, then continue with the Stroop game, and at the end to do all difficult levels of the games.

\section{A. PANAS}

The PANAS questionnaire was filled in before the relaxation part and after the news part. For each session we computed the difference between the mood after the interaction and before the interaction. We wanted to see if there are differences between the morning and evening sessions, and between the interaction days.

For the positive affect difference, an one-way ANOVA analysis shows that the participant felt better after the morning session than after the evening session $(F(1,6)=0.94$, $\mathrm{p}=0.368)$. On days day 2 and day 3 the participant felt better after the interaction than in the last two days (day4, day5) $(F(3,4)=5.43, p=0.067)$. This result can be related to the participant's health. On the last two days he had some health problems and was not feeling good at all.

No differences were found for the negative affect. The negative affect recorded was at a constant value of 10 for 7 out of the entire 8 interaction sessions considered for the analysis. The only day in which the negative affect increased after the experiment, was on day 3 in the

evening session. As seen previously, an increased value for negative affect is associated with unpleasurable engagement.

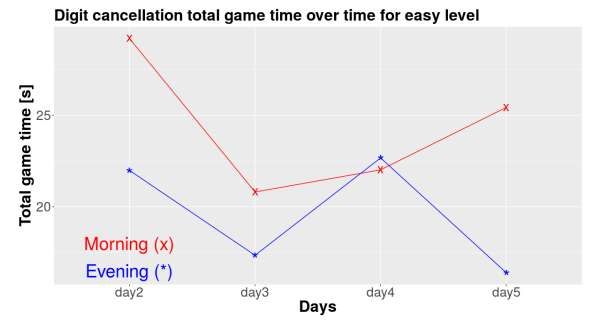

(a)

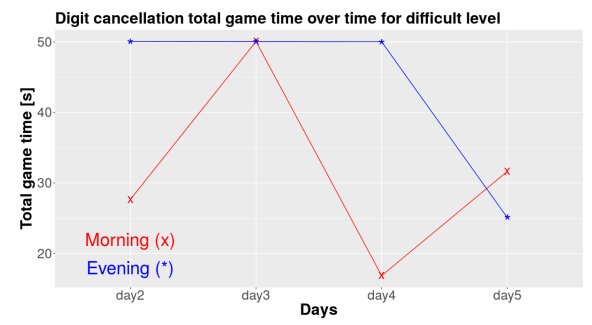

(b)

Fig. 6: Digit Cancellation total game time over time: (a) Easy level; (b) Difficult level

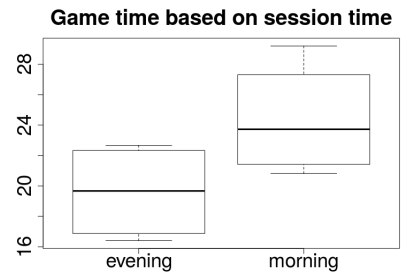

(a)

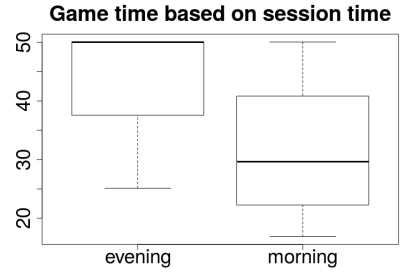

(b)
Fig. 7: Digit Cancellation: (a) Easy level; (b) Difficult level

During that session, for the news reading application multiple technical difficulties were encountered, and the news phase had to be restarted multiple times.

\section{B. Digit Cancellation}

For the Digit Cancellation game three parameters were extracted and used for the analysis: total game time, average time to select an answer, and the total number of mistakes. The two factors used for analysis are the day when the sessions took place (day2, day3, day4, or day5), and the time of the session (morning, or evening). Figure 6a shows the variation of the total game time in each day for the easy level, while Figure 6b shows for the difficult level.

For the total game time, we found that in general our participant finished the easy level faster in the evening session than in the morning one $(F(1,6)=3.72, p=0.1)$ (Figure 7a). While for the difficult level the opposite was found $(\mathrm{F}(1$, 6 ) $=1.73, p=0.23$ ) (Figure 7b). This is also due to the fact that the participant did not finish the difficult level 4 times out of the total 8 times taken into consideration for the analysis. No important differences were found for the total game time between the days.

For the average time to select an answer, we found that 


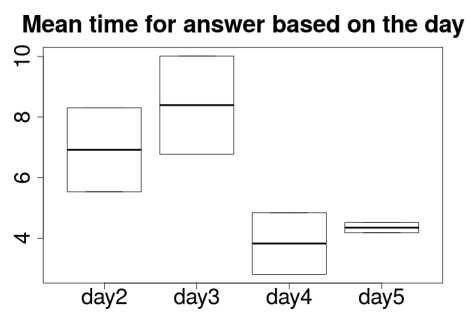

Fig. 8: Digit cancellation average time to make a selection based on the day

for the easy level in the morning hours the participant took longer to make a selection than in the afternoon hours $(\mathrm{F}(1$, $6)=0.96, p=0.36$ ). For the difficult level, the average time to make a selection was similar in the morning and evening sessions.

For the analysis based on the days, we found that for the difficult level, the participant took longer to make a selection in the first two days than in the last two days $(F(3,4)=3.32$, $\mathrm{p}=0.13$ ) (Figure 8). No important results were found for the easy level.

During the easy level the participant made no mistakes. For the difficult level, the participant made the most mistakes in the evening hours $(\mathrm{F}(1,6)=2.359, \mathrm{p}=0.175)$. Moreover, in the first two days more mistakes were made than in the last two days $(\mathrm{F}(1,6)=2.359, \mathrm{p}=0.175)$.

Overall, even if no significant results were found, a tendency can clearly be seen. More interaction sessions and more participants could help find the significant results.

The results of the post questionnaire show that the participant did not find the easy level neither difficult, nor stressing. While for the difficult level, the participant reported that he found the game difficult, but not stressing.

\section{Integer Matrix Task}

As the participant really tried not to make any mistakes, only one mistake was made in all 8 interaction sessions. Therefore, only the number of correctly solved matrices and the time to find the first of the two digits were considered for the analysis. Figure 9a shows the number of correctly solved matrices for each day for the easy level, while Figure $6 \mathrm{~b}$ shows for the difficult level.

For the total number of correctly solved matrices no differences were found for the easy level between the morning and evening sessions. For the difficult level, we found that the participant solved more matrices in the evening than in the morning $(F(1,6)=3.54, p=0.1)$ (see Figure 10).

We found that in the first day the participant performed worse in the easy level than in the other days $(F(3,4)=4.615$, $\mathrm{p}=0.086)$, especially compared to day $3(\mathrm{p}=0.22)$ and day 4 $(\mathrm{p}=0.17)$. No important differences were observed for the difficult level.

When considering the time taken to find the first answer the following results were found. For the difficult level we found that the participant found more quickly the answers in the evening session than in the morning session

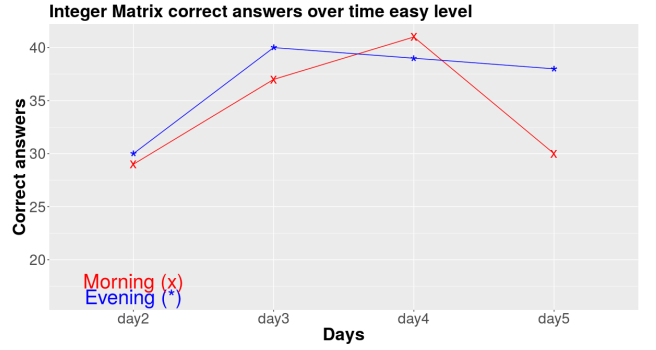

(a)

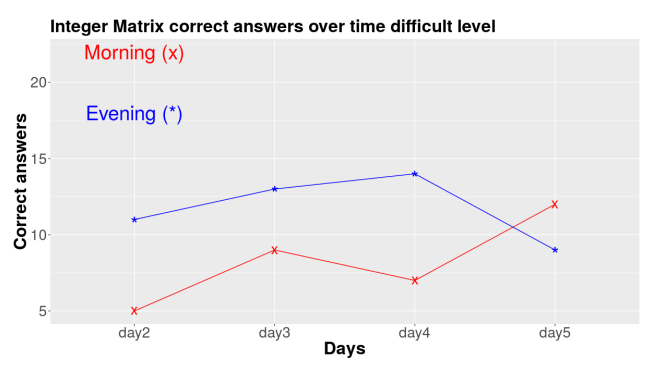

(b)

Fig. 9: Integer Matrix correct answers: (a) Easy level; (b) Difficult level

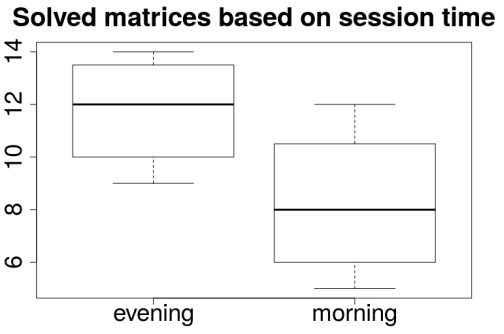

Fig. 10: Matrix task correctly solved matrices in the difficult level based on interaction time

$(F(1,6)=2.315, p=0.17)$. No differences were found for the easy level.

For the analysis based on the day of interaction, for the easy level, it took longer to find the first element in day2 than in any other day $(F(3,4)=5.4, p=0.06)$. For the difficult level, no differences were found between the days.

The participant did not find the easy level difficult, nor stressing, while the difficult level was considered difficult but not stressing.

\section{Stroop game}

For this game we only considered for the analysis the average reaction time and the total game time. Only three mistakes were found throughout the 8 sessions.

No differences were found between the morning and the afternoon sessions for the game time and neither for the average reaction time.

On day 2 it took longer, on average, to find the answer than on the other days $(\mathrm{F}(3,4)=9.69, \mathrm{p}=0.02)$, especially when compared to day3 $(\mathrm{p}=0.056)$, and day5 $(\mathrm{p}=0.052)$. 


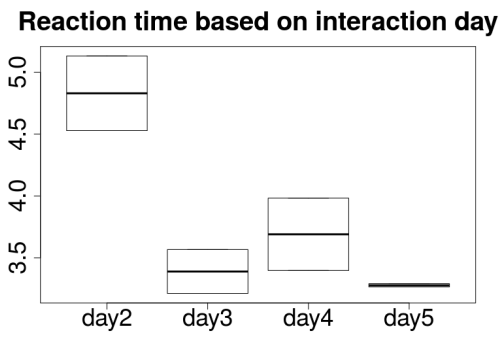

Fig. 11: Stroop Game average reaction time based on interaction day

\section{Discussion}

For this study we only had one participant, therefore, the results that we obtained will have to be confirmed with more participants, including also females. This preliminary data shows that there is a tendency for this participant to perform differently in the morning and in the afternoon hours. Furthermore, we found evidence that there is an improvement over time.

The results from the Digit Cancellation show that the participant finished the game faster in the evening than in the morning for the easy level. No mistakes were made for the easy level, but some mistakes were made for the difficult one. Furthermore, for the difficult level, the participant did not finish the game 4 times out of the total 8 sessions. The number of mistakes decreased from the first two days to the last two days. We believe that with more sessions the participant would improve its performance even more.

Regarding the Integer Matrix Task, for the difficult level the participant solved more matrices in the evening than in the morning, and it took a shorter time to find the answer in the evening than in the morning. For the easy level, the participant showed an improvement after the first day, while for the difficult level, more sessions are needed to see a clear improvement.

A clear improvement over time was found for the Stroop game. After only one day the participant performed significantly better in the following days.

Our extraverted participant was more efficient at around $3 \mathrm{pm}$ than at around $11 \mathrm{am}$, a result which is in accordance with the theory proposed by Colquhoun [23]. For the morningness-eveningness level, we found that only for the Digit Cancellation difficult level the participant performed better in the morning than in the evening. Further studies have to be devised in order to investigate if the same results are found for other elderly with MCI.

One of the questions in the custom developed postquestionnaire asked the participant if he would have preferred to do the same tasks on a tablet. After each interaction he said that he preferred to interact with the robot.

\section{CONCLUSION}

In conclusion, this study represents a first attempt in how the cognitive performance of an individual with mild cognitive impairment varies through an interaction of 5 days. Moreover, it was investigated if there are differences between morning and evening sessions. The results obtained give us a starting point for future studies, in which, more participants could take part in a long-term cognitive stimulation study.

Some of our future work include the analysis of the physiological parameters, to investigate if they are in accordance with the performance results. Furthermore, the data from the News Reading application will be analyzed.

\section{REFERENCES}

[1] W. He, D. Goodkind, and P. Kowal, "An aging world: 2015," International Population Reports, 2016.

[2] D. Feil-Seifer and M. J. Mataric, "Defining socially assistive robotics," in 9th International Conference on Rehabilitation Robotics, 2005.

[3] S. Coradeschi and et al, "Giraffplus: A system for monitoring activities and physiological parameters and promoting social interaction for elderly," Human-Computer Systems Interaction: Background and Applications 3 Advances in Intelligent Systems and Computing, 2014.

[4] K. Papoutsakis and et.al., "Developing visual competencies for socially assistive robots: the hobbit approach," in Proceedings of the 6th International Conference on PErvasive Technologies Related to Assistive Environments. ACM, 2013, p. 56.

[5] G. Stavropoulos and et.al., "Automatic action recognition for assistive robots to support mci patients at home," in Proceedings of the 10th International Conference on PErvasive Technologies Related to Assistive Environments. ACM, 2017.

[6] M. Tanaka, A. Ishii, and et al, "Effect of a human-type communication robot on cognitive function in elderly women living alone," Medical Science Monitor, vol. 18, 2012.

[7] A. Tapus, C. Tapus, and M. J. Mataric, "Music therapist robot for individuals with cognitive impairments," in Proceedings of the ACM/IEEE Human-Robot Interaction Conference (HRI), 2009.

[8] A. Di Nuovo and et.al., "The multi-modal interface of robot-era multirobot services tailored for the elderly," Intelligent Service Robotics, 2017.

[9] R. Agrigoroaie and A. Tapus, "Contactless physiological data analysis for users quality of life improving by using a humanoid social robot,' in 19th Conference on Image Analysis and Processing, 2017.

[10] _ " "Influence of robot's interaction style on performance in a stroop task," in International Conference on Social Robotics, 2017.

[11] J. Pages, L. Marchionni, and F. Ferro, "Tiago: the modular robot that adapts to different research needs," International Workshop on Robot Modularity, IROS, 2016.

[12] H. J. Eysenck and S. B. G. Eysenck, Manual of the Eysenck Personality Questionnaire (junior and adult). Hodder and Stoughton, 1975.

[13] H. Eysenck, "Psychophysiology and personality: Extraversion, neuroticism and psychoticism," Physiological correlates of human behavior, vol. 3, pp. 13-30, 1983.

[14] — The biological basis of personality. Springfield, 1967.

[15] J. Horne and O. stberg, "A self-assessment questionnaire to determine morningness-eveningness in human circadian rhythms," International Journal of Chronobiology, 1976.

[16] P. Corr and A. Cooper, "The reinforcement sensitivity theory of personality questionnaire: Development and validation." Psychological Assessment, 2016.

[17] J. Gray and N. McNaughton, The neuropsychology of anxiety: An enquiry into the functions of the septo-hippocampal system, 2 nd ed. New York: Oxford University Press, 2000.

[18] D. Watson, L. Clark, and A. Tellegan, "The development and validation of brief measures of positive and negative affect: the panas scales," Journal of personality and social psychology, 1988.

[19] D. Watson and L. A. Clark, "Negative affectivity: the disposition to experience aversive emotional states." Psychological bulletin, 1984.

[20] R. Agrigoroaie, D. Ciocirlan, and A. Tapus, "News application adaptation based on user sensory profile," in International Conference on Social Robotics. Springer, 2017, pp. 708-717.

[21] J. Stroop, "Studies of interference in serial verbal reactions," Journal of Exploratory Psychology, 1935.

[22] D. Kahneman, Attention and effort. New Jersey: Prentice-Hall, 1973

[23] W. Colquhoun, "Temperament, inspection efficiency, and time of day," Ergonomics, vol. 3, no. 4, pp. 377-378, 1960. 